DOI 10. 18307/2018. 0412

(C) 2018 by Journal of Lake Sciences

\title{
基于高频次 $\mathrm{GOCl}$ 数据的太湖悬浮物浓度短期动态和驱动力分析”
}

\author{
胡耀躲 ${ }^{1,2}$, 张运林 $^{1 * *}$, 杨 波 $^{2}$, 张毅博 ${ }^{1}$ \\ (1:中国科学院南京地理与湖泊研究所湖泊与环境国家重点实验室,南京 210008) \\ (2: 湖南师范大学资源与环境科学学院 GIS 研究中心, 长沙 410081)
}

\begin{abstract}
摘 要: 总悬浮物是水体中重要的光学敏感物质之一, 很大程度上决定了水柱中光的吸收、散射和衰减, 同时吸附营养 盐、重金属和有毒有害物,对水体物质生物地球化学过程、沉积物埋藏动力和湖泊环境演化具有重要的意义. 基于星地同 步实验和静止水色成像仪 GOCI(Geostationary Ocean Color Imager) 构建了太湖悬浮物浓度估算模型, 并分析了典型风浪过 程中太湖悬浮物浓度短期动态变化过程. 研究表明: 对太湖水体悬浮物浓度较为敏感的波段为 GOCI 的第 7 波段 ( 745 $\mathrm{nm}$ ) 和第 8 波段 $(865 \mathrm{~nm}$ ), 悬浮物浓度与对应波段遥感反射率线性相关决定系数分别为 0.72 和 0.55 ; 基于 GOCI 第 7 波 段的悬浮物浓度单波段遥感估算模型能较为准确地估算太湖的悬浮物浓度, 模型相对均方根误差和平均绝对百分误差 分别为 $28.3 \%$ 和 $24.4 \%$. 通过研究典型风浪过程前后太湖悬浮物浓度变化发现其短期动态变化显著, 风速、风向是悬浮物 浓度短期动态变化的重要驱动因素, 悬浮物浓度与风速呈正比, 并随着风向扩散; 高频连续 GOCI 影像结果显示悬浮物浓 度短期动态变化对风浪扰动的响应有一定的滞后性, 滞后时间为数小时到 1 天, 悬浮物沉降与沉积物再悬浮的临界风速 约为 $3.4 \mathrm{~m} / \mathrm{s}$.
\end{abstract}

关键词: GOCI; 太湖; 总悬浮物;遥感估算; 风浪

\section{Short-term dynamics and driving factors of total suspended matter concentration in Lake Taihu using high frequent geostationary ocean color imager data}

\author{
HU Yaoduo ${ }^{1,2}$, ZHANG Yunlin ${ }^{1 * *},{\text { YANG } \text { Bo }^{2} \& \text { ZHANG Yibo }}^{1}$ \\ (1: State Key Laboratory of Lake Science and Environment, Nanjing Institute of Geography and Limnology, Chinese Academy \\ of Sciences, Nanjing 210008, P.R. China) \\ (2: College of Resources \& Environmental Science, GIS Research Center, Hunan Normal University, Changsha 410081, P.R. \\ China)
}

\begin{abstract}
Total suspended matter (TSM) is one of the important optical active substances in water body, which largely determines the absorption, scattering and attenuation of light in water column and adsorbs nutrients, heavy metals and toxic and harmful substances. Therefore, TSM is of great important to biogeochemical processes of water substance, lake sediment burial dynamics andlake environment evolution. This study developed TSM estimation model in Lake Taihu based on satellite-ground synchronous experiment and Geostationary Ocean Color Imager ( GOCI) image, and further analyzed TSM short-term dynamics in Lake Taihu during its typical wind-waves process. The results showed that the band $7(745 \mathrm{~nm})$ and band $8(865 \mathrm{~nm})$ of GOCI data were the sensitive bands of TSM estimation in Lake Taihu with the linear correlation determination coefficients of 0.72 and 0.55 , respectively. A single band empirical model based on GOCI band 7 was used to estimate the TSM concentration of Lake Taihu and its relative root mean square error and mean absolute percentage error were $28.3 \%$ and $24.4 \%$, respectively. Significant short-term TSM dynamic change was observed in Lake Taihu, and wind direction and wind speed were the important factors determining TSM concentration. TSM concentration increased with the increase of wind speed and diffused along with wind direction. High-frequency continuous GOCI images showed that the short-term dynamics of TSM concentration had a certain lag in response to wind-wave disturbance. The lag time ranged from several hours to one day, and the critical wind speed of TSM settling and sediment resuspension was about
\end{abstract}

* 国家自然科学基金项目 $(41621002,41661134036)$ 和中国科学院前沿科学重点研究项目 ( QYZDB-SSW-DQC016) 联 合资助. 2017-10-08 收稿; 2017-11-13 收修改稿. 胡耀躲 (1993 ), 男, 硕士研究生; E-mail: glashyd@ yeah.net.

** 通信作者;E-mail:ylzhang@ niglas.ac.cn. 
$3.4 \mathrm{~m} / \mathrm{s}$.

Keywords: GOCI; Lake Taihu; total suspended matter; remote sensing estimation; wind-waves

总悬浮物 (total suspended matter, TSM) 是水体中重要的光学敏感物质之一, 对光具有强吸收和强散射的 特性, 因此水体中的悬浮物浓度直接决定着水体浑浊度、水色和透明度等光学性质,进而影响湖泊水体的初 级生产力和生态系统类型 ${ }^{[1-2]}$. 同时, 悬浮物含有大量的颗粒态营养盐, 吸附和附着各类重金属和有毒有害 物等, 严重影响到营养盐、重金属和有毒有害物的迁移、降解和转化过程 ${ }^{[3]}$. 过去 25 年 (1992-2016 年), 关 于悬浮物的研究呈现快速增长态势, 在 Web of Science 数据库中近年来每年新增 1000 篇左右相关 SCI 论文 (图 1). 传统的悬浮物浓度估算主要依赖野外实测和采样分析得到,这种方法工作量大,效率低,而且由于 采样点的局限性, 导致实验结果难以反映整个区域的水质情况, 从而难以实现水体悬浮物浓度的同步动态 监测和精细化的空间分布描述. 近年来传感器在线监测技术快速发展, 通过浊度等探头参数的转换能够实 现单一站点悬浮物浓度的动态变化过程实时在线监测 ${ }^{[4]}$,但在线监测站点有限,仍无法弥补空间过程的同 步动态监测, 不能有效地揭示悬浮物浓度时空格局的精细化过程. 遥感数据本身具有获取数据快, 覆盖范围 广和同步观测的优势, 基于遥感数据的悬浮物浓度监测能够反映不同时空尺度的浓度动态变化过程 ${ }^{[5]}$.

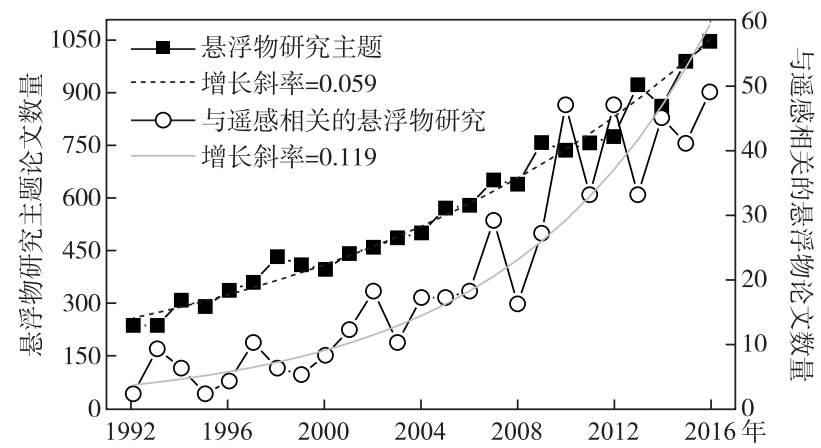

图 $1 \mathrm{Web}$ of Science 数据库中检索悬浮物以及与遥感相关的悬浮物研究论文数量随时间 (1992-2016 年)

变化趋势 $($ 检索策略: TS $=($ “suspended matter * ” or “suspended $\operatorname{solid} *$ ” or

“suspended particle matter $*$ ”) 和 TS $=$ ( “ suspended matter $*$ ” or “ suspended $\operatorname{solid} *$ ” or "suspended particle matter *") and TS= "remote sensing")

Fig.1 Trends of total publication searching suspended matter and remote sensing-related suspended matter in the Web of Science database over time (1992-2016)

利用遥感方法开展悬浮物浓度的遥感监测、动态变化过程和驱动机制的研究尽管在整个悬浮物领域的 研究中占比很少, 但也由来已久, 近年来更是呈现出更快速的增长速率 (图 1). 如与遥感相关的悬浮物研究 SCI 论文指数函数增长率是整个悬浮物研究 SCI 论文指数函数增长率的近 2 倍 (图 1). 当前, 许多学者利用 MODIS、MERIS、Landsat TM/ETM/OLI 和 SeaWiFS 等遥感数据分别构建了悬浮物浓度反演方法并进行应用. Shi 等 ${ }^{[6]}$ 发现原位悬浮物浓度数据和大气校正后 MODIS 在 $645 \mathrm{~nm}$ 波段的遥感反射率 $\left(R_{\mathrm{rs}}(645)\right)$ 之间具有 显著相关关系 $\left(R^{2}=0.70, P<0.001, n=150\right)$, 并基于经验模型估算了太湖悬浮物浓度, 发现太湖悬浮物浓度 具有明显的季节分布规律, 夏季和秋季的悬浮物浓度明显低于春季和冬季. Zhang 等 ${ }^{[7]}$ 利用 MERIS 数据的 第 9 波段建立了太湖悬浮物浓度估算的经验模型 $\left(R^{2}=0.72, P<0.001, n=73\right)$, 模型的相对均方根误差和平 均相对误差分别为 $36.9 \%$ 和 $31.6 \%$, 结果显示太湖的日均风速与悬浮物浓度有显著的相关性, 并且有沉水植 被的区域悬浮物浓度较低. Zheng 等 ${ }^{[8]}$ 开发了一种基于 Landsat 影像近红外波段的悬浮物浓度估算算法,估 算了 1978- 2013 年洞庭湖的悬浮物浓度, 研究发现水位和七天的累计降雨量与悬浮物浓度呈显著相关. Nechad 等 ${ }^{[9]}$ 对一种多传感器 (SeaWIFS、MERIS 和 MODIS) 通用的悬浮物浓度反演算法进行了校准和验证, 结果显示在光谱 670 750 nm 范围应用非线性回归分析的校准数据集估算悬浮物浓度的误差在 30\%以下, 
通过单点时间序列和单个影像证明了总悬浮物反演算法在多传感器环境下的一致性. 以上学者利用不同卫 星反演了不同湖泊的悬浮物浓度, 并取得了较好的结果, 但以上卫星由于时间分辨率较低, 难以及时捕捉浅 水湖泊悬浮物浓度短期动态变化过程, 对于悬浮物浓度快速变化的驱动机制和可能引发的一些污染过程不 能形成有效监测及预警, 实用性受到很大的限制 ${ }^{[10]}$. 系统研究风浪扰动下太湖悬浮物浓度的短期动态变化 规律, 有助于整体把握太湖水质的变化, 探求水动力过程对沉积物再悬浮、底泥营养盐释放和蓝藻水华暴发 的影响机制, 为太湖的蓝藻水华灾害和湖泛等预测预警提供数据支撑和决策依据 ${ }^{[1]}$.

静止水色成像仪 GOCI ( Geostationary Ocean Color Imager) 是韩国于 2010 年 6 月 27 日发射的搭载在全球 第一颗多用途静止轨道卫星上的传感器, 2011 年 4 月开始提供数据, 其主要目的是从静止的平台上以高时 间频率持续观测环朝鲜半岛的海洋环境并提供渔业信息 ${ }^{[12]}$. 相比于其他水色卫星, GOCI一天能获取八景 遥感影像, 增加了数据获取的机会, 大大降低了云层干扰造成误差和数据缺失的概率, 其数据有着更高的时 间分辨率以及信噪比 ${ }^{[13]}$. GOCI 数据已广泛应用于近岸和湖泊水体悬浮物浓度估算及动态监测, 并被证明 是一种极具潜力的悬浮物遥感监测手段 ${ }^{[1-15]}$. 但已有的研究大多基于 GOCI 数据开展模型构建, 悬浮物浓度 遥感估算和时空格局, 较少涉及悬浮物浓度短期动态变化过程及驱动力分析. 此外, 已有的经验模型星地同 步实验实测悬浮物浓度数据较少且集中在河口海岸和近海, 模型应用到太湖等高浑浊的富营养化浅水湖泊 尚存在一定的不确定性. 因此, 本研究基于在太湖开展的大量星地同步实验, 首先构建和校验了基于 GOCI 传感器影像数据的悬浮物浓度遥感估算模型, 基于高频次的 GOCI 观测数据重点探讨其短期动态变化, 分析 典型风浪过程影响下太湖悬浮物浓度的迁移规律.

\section{1 数据与方法}

\section{1 研究区概况}

太湖 ( $30^{\circ} 56^{\prime} \sim 31^{\circ} 34^{\prime} \mathrm{N}, 119^{\circ} 54^{\prime} \sim 120^{\circ} 36^{\prime} \mathrm{E}$ ) 位于长江三角洲的南缘, 是中国五大淡水湖之一, 水面面积 $2338 \mathrm{~km}^{2}$, 平均水深 $2 \mathrm{~m}$, 是一个典型的大型浅水富营养化湖泊 ${ }^{[16]}$. 太湖自东向西有东太湖、胥口湾、贡湖 湾、梅梁湾和竺山湾 5 个湖湾 (图 2). 太湖作为重要的内陆水源地, 其水质变化与人类活动关系密切 ${ }^{[17]}$. 太 湖在风浪作用下, 沉积物极容易发生再悬浮, 沉积物再悬浮不仅增加了水体中的悬浮物浓度, 同时释放营养 盐, 加剧了太湖的富营养化 ${ }^{[18]}$, 因此监测太湖水体中悬浮物浓度动态变化过程, 揭示其驱动机制对太湖水环 境监测和蓝藻水华控制和湖泊富营养化治理都具有重要意义.
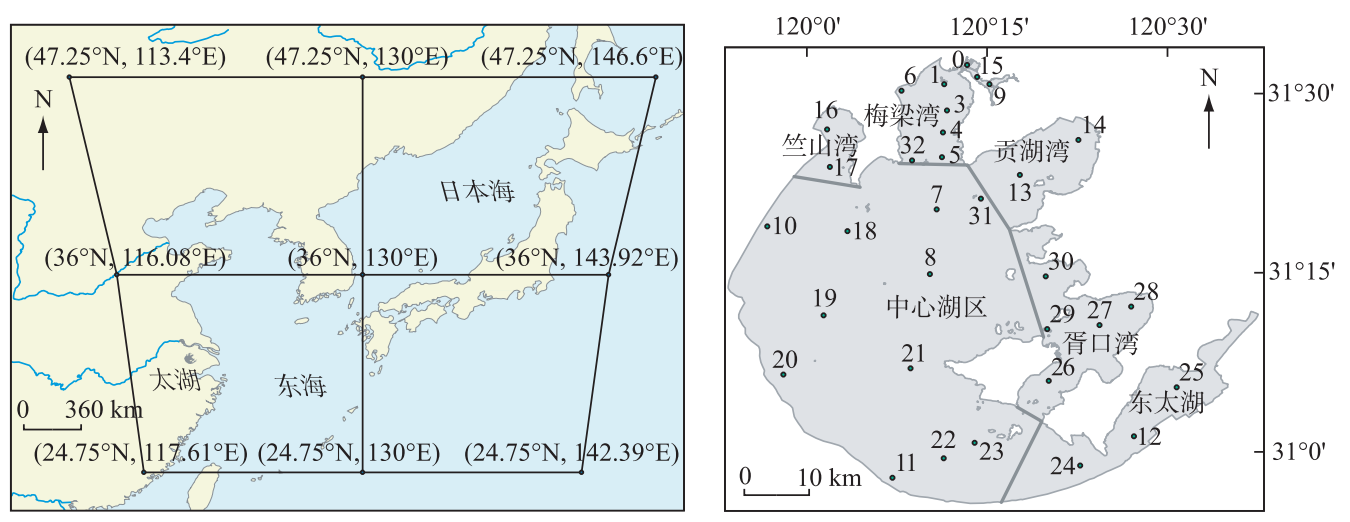

图 2 GOCI 成像范围及太湖采样点分布

Fig.2 GOCI imaging region and distribution of sampling sites in Lake Taihu

\section{2 野外采样与室内分析}

$2012-2015$ 年在太湖共进行 8 次星地同步遥感实验, 其中 $2 、 5 、 8$ 及 11 月份采集全湖各点位, 其余月份 只采集北部湖区的点位 (包括 $0 、 1 、 3 \sim 8 、 10 、 13 、 14 、 16 、 17$ 和 32 样点) (图 2), 除去东太湖及异常点后共获得 130 个实测悬浮物浓度数据 (表 1). 悬浮物 ( TSM) 浓度采用 GB 11901-1989 标准测定, 即将孔径为 $0.70 \mu \mathrm{m}$ 
的 Whatman $\mathrm{GF} / \mathrm{F}$ 滤膜用马福炉在 $450^{\circ} \mathrm{C}$ 温度下煅烧 $4 \mathrm{~h}$, 用高精度电子秤称重得到空白膜的重量, 然后过滤 一定体积的水样在 $105^{\circ} \mathrm{C}$ 温度下烘干再称重,进而计算得到悬浮物浓度 ${ }^{[6]}$.

表 1 2012－2015 年星地同步实验基本情况

Tab.1 Information of satellite-ground synchronous experiment from 2012 to 2015

\begin{tabular}{cccccc}
\hline 采样时间 & 站点分布 & 样品数 & TSM 范围 $/(\mathrm{mg} / \mathrm{L})$ & TSM 均值 $/(\mathrm{mg} / \mathrm{L})$ & GOCI 卫星过境时间 \\
\hline $2012-05-16$ & 北部湖区 & 32 & $6.5 \sim 71.1$ & $30.1 \pm 19.5$ & $09: 28$ \\
$2012-07-25$ & 北部湖区 & 14 & $28.5 \sim 242.1$ & $95.3 \pm 48.6$ & $09: 28$ \\
$2012-11-14$ & 全湖 & 32 & $6.5 \sim 167.5$ & $57.9 \pm 43.2$ & $08: 28$ \\
$2013-05-14$ & 全湖 & 32 & $10.9 \sim 252.1$ & $60.3 \pm 52.0$ & $09: 28$ \\
$2013-07-17$ & 北部湖区 & 14 & $27.3 \sim 59.2$ & $46.0 \pm 9.4$ & $14: 28$ \\
$2014-03-18$ & 北部湖区 & 14 & $7.5 \sim 57.2$ & $18.8 \pm 12.1$ & $08: 28$ \\
$2014-10-14$ & 北部湖区 & 14 & $18.4 \sim 111.0$ & $62.6 \pm 30.7$ & $08: 28$ \\
$2015-12-17$ & 北部湖区 & 14 & $16.9 \sim 129.1$ & $36.9 \pm 35.0$ & $10: 28$ \\
\hline
\end{tabular}

\section{$1.3 \mathrm{GOCl}$ 影像数据及气象数据}

本研究使用的是 2012-2015 年获取的与采样当天相匹配的 8 景 GOCI L1B 影像, 其中 GOCI 影像来自 韩国海洋技术研究院 (http://kosc. kiost.ac/). 基于 Landsat-8 OLI 对 GOCI 影像进行几何校正, 然后使用 ENVI 软件进行辐射定标, 将 $D N$ (Digital Number) 值转化为大气顶层的遥感反射率, 接着利用 $6 \mathrm{~S}^{[19]}$ ( Second Simulation of the Satellite Signal in the Solar Spectrum) 辐射传输模型进行大气校正, 目的是为了消除大气和光 照等因素对地物反射的影响,最后利用太湖的矢量边界进行研究区域裁剪.

风速、风向数据来源于中国气象数据共享网 ( http://cdc.nmic.cn), 其中探讨竺山湾悬浮物浓度使用的 是临近的宜兴气象监测点数据, 探讨梅梁湾、贡湖湾悬浮物浓度动态变化使用的是临近的无锡气象监测点 数据, 而探讨胥口湾悬浮物浓度动态变化使用临近的苏州东山气象监测点数据.

\section{4 数据分析与处理}

利用 MATLAB 对 130 组 GOCI 影像遥感反射率中自动选取 105 组数据与相对应点位的实测悬浮物浓度 进行相关性分析, 并用余下 25 组数据进行检验.

遴选 2012 年 7 月 21-26 日为期 6 天连续天气晴好有 GOCI 影像的典型风浪过程进行分析,利用公式 (3) 反演得到的 2012 年 7 月 21-26 日的日平均悬浮物浓度分布,对其取平均值得到当天悬浮物浓度分布, 进而进行分析. 由于太湖东南部的东太湖属于水草覆盖区域,估算结果误差很大, 故对此水草区域进行掩膜.

运用 SPSS 软件进行数据统计分析, 计算平均值、绝对值、标准差、方差分析等,显著性水平用显著 $(P \leqslant$ $0.05)$ 和不显著 $(P>0.05)$ 表示. 模型系数的确定在 Matlab 软件中采用最小二乘法非线性拟合得到. 本研究 引人决定系数 $\left(R^{2}\right) 、 F$ 值、平均绝对百分比误差 ( mean absolute percentage error, $M A P E$ ) 以及相对均方根误差 (relative root mean square error, RRMSE) 作为统计量来检验估算模型的适用性. RRMSE 及 $M A P E$ 的计算公式 如下:

$$
\begin{aligned}
& \text { RRMSE }=\sqrt{\frac{\sum_{i=1}^{n}\left(\frac{x_{i \mathrm{Mea}}-x_{i \mathrm{Est}}}{x_{i \mathrm{Mea}}}\right)^{2}}{n}} \times 100 \% \\
& M A P E=\frac{\sum_{i=1}^{n}\left(\frac{\left|x_{i \mathrm{Mea}}-x_{i \mathrm{Est}}\right|}{x_{i \mathrm{Mea}}}\right)}{n} \times 100 \%
\end{aligned}
$$

式中, $x_{i \mathrm{Mea}}$ 为实测值, $x_{i \mathrm{Est}}$ 为第 $i$ 波长的模拟值, $n$ 为样本数.

\section{2 结果与分析}

\section{$2.1 \mathrm{GOCl}$ 数据大气校正结果对比}

利用 $6 \mathrm{~S}$ 辐射传输模型对 GOCI 进行大气校正得到了广泛应用, 其大气校正效果得到了普遍认可. 根据 
姜戬等 ${ }^{[20]}$ 对太湖的研究, 发现 2012 年太湖的平均能见度为 $15 \mathrm{~km}$ 左右, 当能见度大于 $20 \mathrm{~km}$ 时, 校正结果 受能见度或者气溶胶的影响较低; 而太湖被苏州、无锡等城市包围, 故气溶胶模型选择为城市型, 能见度设 置为 $15 \mathrm{~km}$. 选取 2013 年 8月 5-6 日的 GOCI 影像, 进行大气校正后对其第 7 波段 (745 nm) 及第 8 波段 (865 nm) 的遥感反射率与实测遥感反射率进行对比和相关分析 (图 3), 发现 $745 \mathrm{~nm}$ 和 $865 \mathrm{~nm}$ 实测遥感反 射率和大气校正后遥感反射率能很好的一一对应, 线性相关可决定系数分别为 0.69 和 $0.67 ; R R M S E$ 分别为 $36.6 \%$ 和 $46.2 \%$, 校正结果较为可靠, 经大气校正后的遥感反射率可以用于悬浮物遥感模型构建与应用.
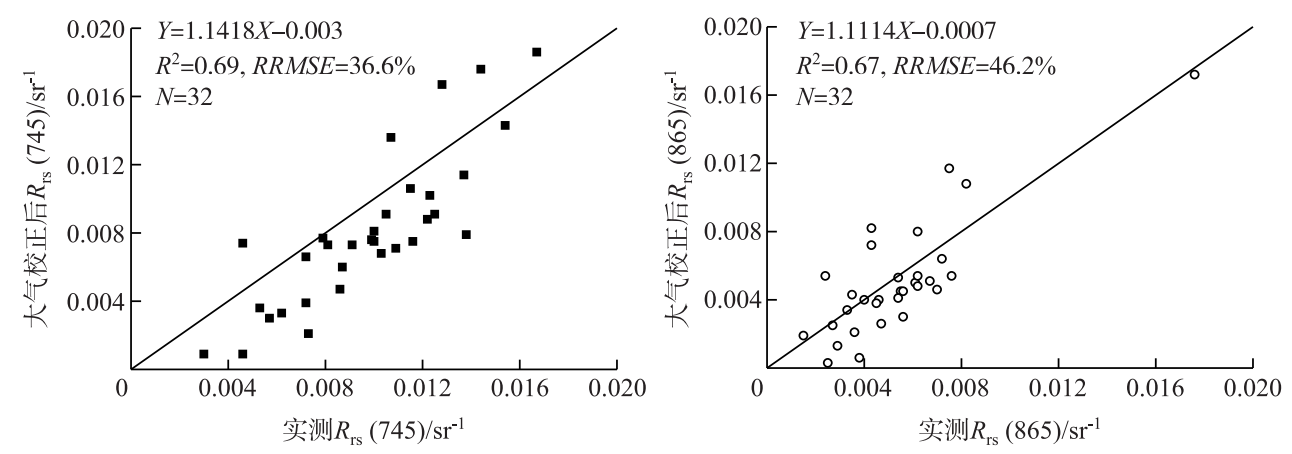

图 3 实测和大气校正后遥感反射率对比

Fig. 3 Comparison of measured and calculated remote sensing reflectance through atmospheric correction

\section{2 敏感波段分析和估算模型构建}

通过 MATLAB 的相关性分析(表 2), 发现对悬浮物浓度比较敏感的波段为第 7 波段(近红外波段, 中心 波长为 $745 \mathrm{~nm}$ ), 决定系数为 0.72 , 其次是第 8 波段 (近红外波段, 中心波长为 $865 \mathrm{~nm}$ ) 和第 6 波段 (红光波 段, 中心波长为 $680 \mathrm{~nm}$ ), 决定系数分别为 0.55 和 0.25 .

表 2 悬浮物浓度单波段估算模型误差统计和模型参数

Tab.2 Single band estimation models error statistics and its parameters

\begin{tabular}{ccccccccc}
\hline 参数 & 波段 1 & 波段 2 & 波段 3 & 波段 4 & 波段 5 & 波段 6 & 波段 7 & 波段 8 \\
\hline 斜率 & -19.1 & 27.2 & 84.2 & 189.7 & 248.9 & 258.6 & 1052.4 & 1411.2 \\
截距 & 43.41 & 39.48 & 32.80 & 17.64 & 17.11 & 12.17 & 1.52 & 8.26 \\
$R^{2}$ & 0 & 0 & 0.02 & 0.08 & 0.15 & 0.25 & 0.72 & 0.55 \\
$F$ & 0.06 & 0.13 & 1.73 & 8.59 & 18.73 & 34.40 & 266.89 & 126.26 \\
$R R M S E$ & $45.7 \%$ & $46.6 \%$ & $47.2 \%$ & $48.2 \%$ & $46.8 \%$ & $49.7 \%$ & $28.3 \%$ & $31.9 \%$ \\
$M A P E$ & $57.9 \%$ & $58.7 \%$ & $58.2 \%$ & $56.7 \%$ & $50.4 \%$ & $50.4 \%$ & $24.4 \%$ & $28.5 \%$ \\
\hline
\end{tabular}

利用实测悬浮物数据与 GOCI 各波段遥感反射率值进行相关性分析, 选取相关性高的波段建立单波段 模型, 通过模型的精度对比、误差分析, 选取最适合的悬浮物反演模型应用于 GOCI 影像上, 继而分析典型风 浪影响下的太湖悬浮物时空变化规律. 表 2 给出了各单波段模型的回归参数、决定系数及检验值, 利用 GOCI 的第 7 波段以及第 8 波段的遥感反射率与实测值进行回归分析, GOCI 第 7 波段遥感反射率与实测值 的散点图见图 $4 \mathrm{a}$, 第 8 波段遥感反射率与实测值的散点图见图 $4 \mathrm{~b}$, 发现散点间具有良好的线性相关关系, $R^{2}$ 分别为 0.72 和 0.55 , 说明 GOCI 的第 7 波段和第 8 波段都能较好地用来估算太湖悬浮物浓度.

利用剩余的验证样本分别对这两个单波段模型进行检验, GOCI 第 7 波段模型的 $F$ 检验值为 266.89 , $R R M S E$ 为 $28.3 \%, M A P E$ 为 $24.4 \%$, 见图 $5 \mathrm{a}$; 第 8 波段的 $F$ 检验值为 $126.26, R R M S E$ 为 $31.9 \%, M A P E$ 为 $28.5 \%$, 见图 5b. 研究表明, GOCI 影像的 8 个波段中对悬浮物最为敏感的波段为近红外波段,赵丽娜等 ${ }^{[21]}$ 的 研究表明, GOCI 的近红外波段(Band 7 和 Band 8) 是浑浊水体悬浮物浓度反演最为有效的波段区间. Choi 等 ${ }^{[22]}$ 利用 GOCI 传感器的第 5 波段 $(660 \mathrm{~nm})$ 建立了估算木浦沿海地区以及京畿湾 TSM 浓度的单波段经验 

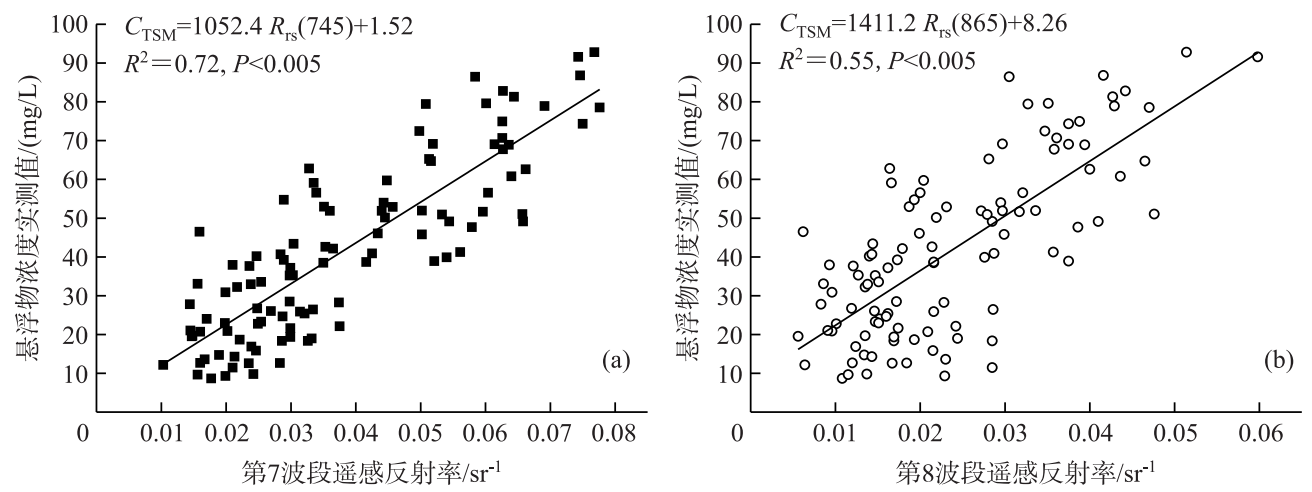

图 4 悬浮物浓度实测值与遥感反射率的回归分析

Fig.4 Regression analysis between measured total suspended matter concentrations and remote sensing reflectance

模型, $R^{2}$ 为 0.92 ; 何贤强等 ${ }^{[14]}$ 利用 GOCI 传感器的第 7 波段 $(745 \mathrm{~nm}$ ) 与第 3 波段 $(490 \mathrm{~nm}$ ) 的比值建立了估 算长江口及杭州湾 TSM 浓度的经验模型, 相关系数为 0.94 ,标准差为 $0.22 \mathrm{mg} / \mathrm{L}$. 以上结果表明基于 GOCI 传感器的经验模型能较好地估算悬浮物浓度. 本文旨在利用相对可靠的反演结果研究风浪扰动下太湖悬浮 物浓度的短期动态变化规律, 利用时间跨度为 4 年的 130 个实测数据建立太湖悬浮物浓度经验估算模型, 结 果表明经验模型能较好地估算太湖的悬浮物浓度 (图 4、图 5). 由于 GOCI 传感器第 7 波段建立的经验模型 的可决系数高于第 8 波段,样本的检验误差小于第 8 波段,本研究将采用第 7 波段建立经验模型来估算悬浮 物浓度,悬浮物的估算公式如下：

$$
C_{\mathrm{TSM}}=1052.4 R_{\mathrm{rs}}(745)+1.52 \quad\left(R^{2}=0.72, n=105, P<0.005\right)
$$

式中, $R_{\mathrm{rs}}(745)$ 为 GOCI 影像第 7 波段的遥感反射率.
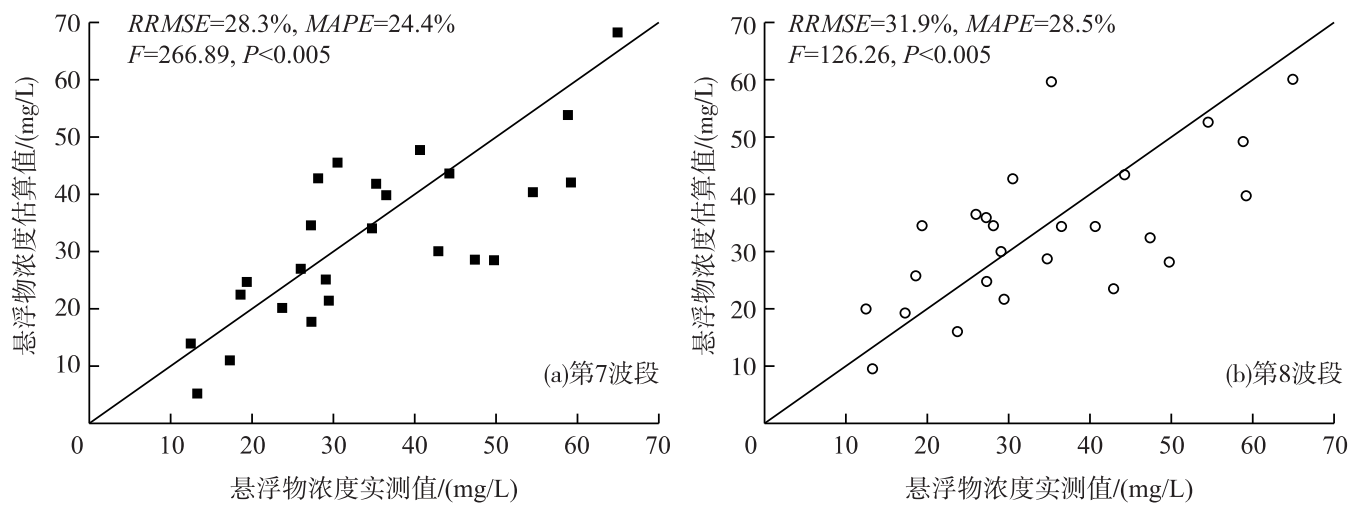

图 5 悬浮物浓度实测值与模型的估算值对比

Fig.5 Comparison between the measured and estimated TSM concentrations

\section{3 典型风浪过程太湖悬浮物浓度变化规律}

图 6 为利用公式 (3) 反演得到的 2012 年 7 月 21-26 日的日平均悬浮物浓度分布,对其取平均值得到当 天悬浮物浓度分布. 太湖东部的胥口湾悬浮物浓度变化不大,但其他区域的悬浮物浓度日间变化较大,除岸 边的水华区域外, 21 和 22 日太湖西北部的竺山湾及沿岸的悬浮物浓度介于 $60 \sim 70 \mathrm{mg} / \mathrm{L}$ 之间, 中心湖区介 于 $40 \sim 50 \mathrm{mg} / \mathrm{L}$ 之间; 至 23 日西北部的竺山湾及沿岸的悬浮物浓度上升到 $80 \sim 120 \mathrm{mg} / \mathrm{L}$, 中心湖区达到 $50 \sim 70 \mathrm{mg} / \mathrm{L}$; 至 24 日太湖西北部的竺山湾及沿岸和中心湖区整体达到 $50 \sim 60 \mathrm{mg} / \mathrm{L} ; 25$ 和 26 日太湖整体的 悬浮物浓度在 24 日的基础上有从西北向东南扩散的趋势, 西北部竺山湾及沿岸的悬浮物浓度介于 $70 \sim 80$ 

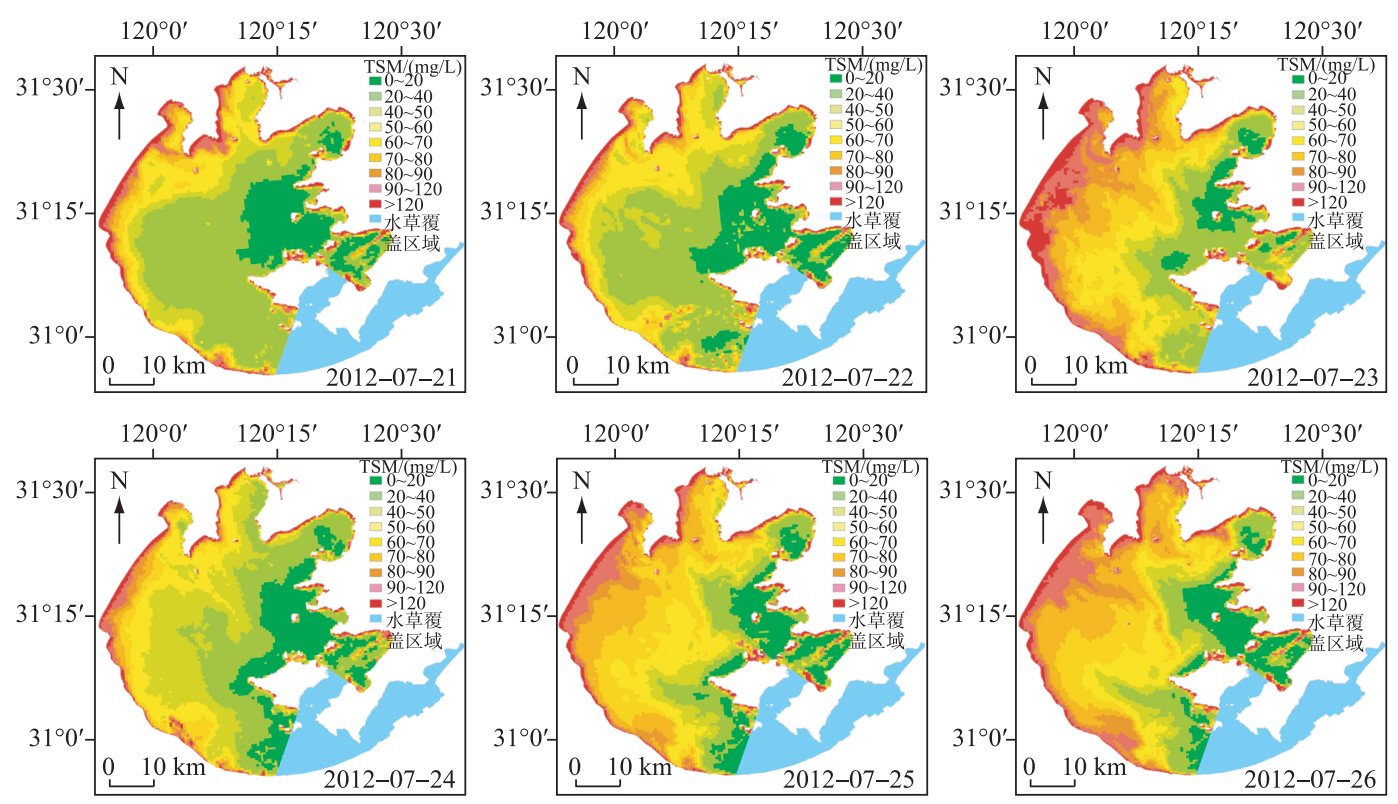

图 62012 年 7 月 $21-26$ 日日均悬浮物浓度空间分布

Fig.6 Spatial distribution of daily TSM concentration from 21 to 26 July 2012

$\mathrm{mg} / \mathrm{L}$ 以及 $80 \sim 90 \mathrm{mg} / \mathrm{L}$ 范围的区域都有所增大, 而中心湖区及东部胥口湾悬浮物浓度变化较小, 中心湖区 的悬浮物浓度介于 $60 \sim 70 \mathrm{mg} / \mathrm{L}$ 之间 (图 6). 整体来说从 7 月 21 日至 26 日太湖竺山湾及沿岸的悬浮物浓 度整体高于太湖其他区域, 并有从西北向东南递减的趋势. 将太湖的悬浮物浓度以 $20 、 40 、 70$ 和 $90 \mathrm{mg} / \mathrm{L}$ 为 间断值分为 $0 \sim 20 、 20 \sim 40 、 40 \sim 70 、 70 \sim 90$ 和 $>90 \mathrm{mg} / \mathrm{L}$ 五级, 图 7 为太湖 2012 年 7 月 21-26 日各个级别悬 浮物浓度面积占比的变化, 其中 $21-26$ 日浓度 $>90 \mathrm{mg} / \mathrm{L}$ 的区域面积所占比分别为 $16.2 \% 、 13.3 \% 、 25.7 \%$ 、 $11.4 \% 、 18.0 \%$ 和 $20.8 \%$. 太湖悬浮物浓度从 7 月 21 日至 23 日逐渐上升, 至 23 日太湖悬浮物浓度 $>70 \mathrm{mg} / \mathrm{L}$ 的区域面积所占比达到 $45.7 \%, 7$ 月 23- 24 日太湖悬浮物浓度介于 $70 \sim 90 \mathrm{mg} / \mathrm{L}$ 以及 $>90 \mathrm{mg} / \mathrm{L}$ 的区域面积 都有所减小, 从 7 月 24- 26 日太湖悬浮物浓度介于 70 90 mg/L 以及 $>90 \mathrm{mg} / \mathrm{L}$ 的区域面积又逐渐上升.

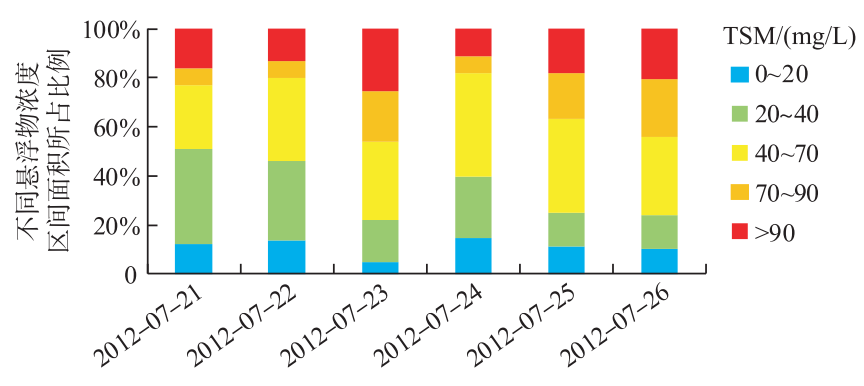

图 7 太湖不同悬浮物浓度区间面积所占百分比变化

Fig.7 Area proportions under different TSM concentration grades in Lake Taihu

2012 年 7 月 21 日的悬浮物浓度日内变化图表明,一天内太湖的悬浮物浓度在整个湖心区域及东南部 分变化较小,且悬浮物浓度维持在一个较低的水平; 而在太湖西北部的竺山湾及沿岸, 水体中悬浮物浓度维 持在一个较高的水平, 浓度大于 $80 \mathrm{mg} / \mathrm{L}$ 的区域从早到晚呈现递减的趋势, 至 $15: 28$ 太湖西北部䇥山湾及沿 岸的悬浮物浓度达到日内最低( 图 8). 


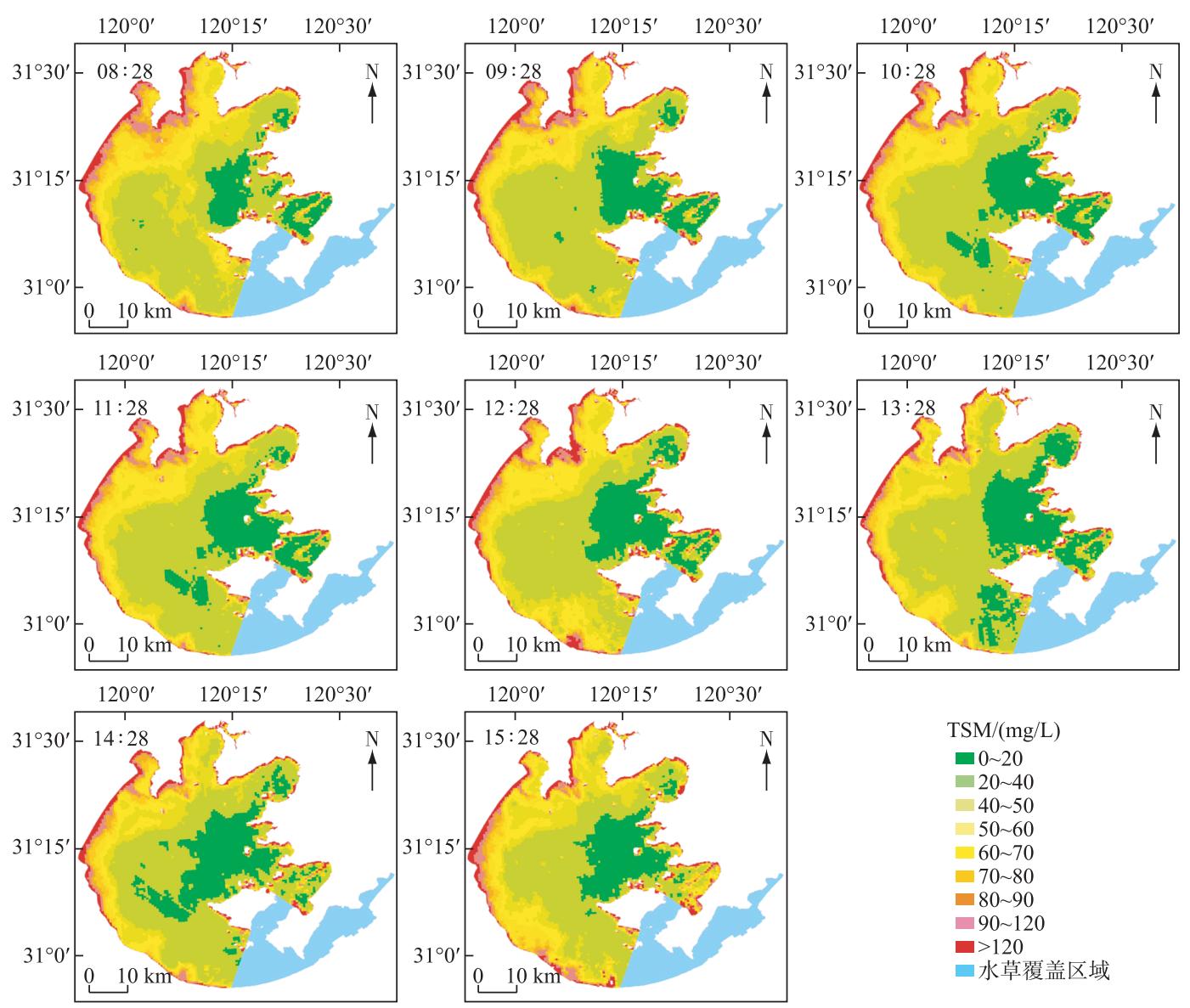

图 82012 年 7 月 21 日太湖悬浮物浓度分布

Fig. 8 Distribution of TSM concentration in Lake Taihu on July 21,2012

\section{3 讨论}

有学者利用 MODIS 影像研究风浪对湖泊悬浮物浓度变化的影响, 发现风速与悬浮物浓度存在着明显的 正相关, 悬浮物浓度的变化对风浪扰动的响应有一定的滞后性 ${ }^{[6-7]}$. 然而一天内的风速变化较大, 一天一景 的 MODIS 影像无法精确评估一天内湖泊的平均悬浮物浓度. COMS 卫星提供得到一天八景 GOCI 影像, 能 较为精确的评估一天内湖泊的平均悬浮物浓度, 并且可以进一步研究风浪过程中悬浮物浓度的逐时变 化 $^{[14,22]}$. 朱广伟等 ${ }^{[11,23]}$ 观测分析了太湖强风浪过程及连续多天的弱风浪过程中总悬浮物浓度变化,结果表 明与弱风浪期间相比, 强风浪期间湖水中的悬浮物浓度提高了 10 倍, 表明风浪扰动是太湖悬浮物浓度变化 的主要原因 ${ }^{[24]}$. 太湖各湾区 (除东太湖) 的平均悬浮物浓度及相应气象监测点的风速变化可以看出, 太湖各 湾区的悬浮物浓度与前一天的风速都有较一致的变化趋势, 说明太湖悬浮物浓度变化对风浪的响应需要一 定时间, 可能存在一定的滞后性, 滞后时间为数小时到 1 天( 图 9). 风浪前 2012 年 7 月 21 日和 22 日的风速 增加, 而 7 月 22 日的悬浮物浓度降低, 说明悬浮物在水中有一个缓慢的沉降过程 ${ }^{[25]}$. 统计 4 个湾区 7 月 21 日的平均风速, 结果为 $3.4 \mathrm{~m} / \mathrm{s}$, 当风速小于 $3.4 \mathrm{~m} / \mathrm{s}$ 时太湖各湾区的悬浮物浓度都有所降低, 此时太湖悬浮 物的沉降速度大于风浪再悬浮; 当风速大于 $3.4 \mathrm{~m} / \mathrm{s}$ 时各湾区的悬浮物浓度逐渐升高, 此时太湖沉积物再悬 浮的速度大于悬浮物的沉降速度, 说明太湖沉积物再悬浮大于沉降的临界风速约为 $3.4 \mathrm{~m} / \mathrm{s}$ (图 9 ). 过去许 多野外原位观测、高频监测、室内模拟和数值模拟结果均显示 $3 \sim 4 \mathrm{~m} / \mathrm{s}$ 的风速是太湖沉积物再悬浮的临界 
风速. 如 Qin 等 ${ }^{[26]}$ 对太湖不同湖区 5 个站点开展的原位观测发现太湖沉积物再悬浮的临界风速为 $4.0 \mathrm{~m} / \mathrm{s}$, $\mathrm{Li}$ 等 ${ }^{[27]}$ 在梅梁湾口高频监测站点进行的连续 11 天观测证实了 $4.0 \mathrm{~m} / \mathrm{s}$ 这样一个临界风速. Wang 等 $^{[28]}$ 的研 究显示太湖沉积物再悬浮的临界风速为 $3 \sim 4 \mathrm{~m} / \mathrm{s}$, 风速达到 $3 \mathrm{~m} / \mathrm{s}$ 时沉积物开始发生再悬浮, 而 Zheng 等 ${ }^{[29]}$ 的研究也表明太湖沉积物再悬浮的临界风速为 $3.7 \sim 4.0 \mathrm{~m} / \mathrm{s}$. 以上结果也进一步证实了利用 GOCI 能够动态 监测风浪过程中太湖悬浮物沉降和再悬浮过程.
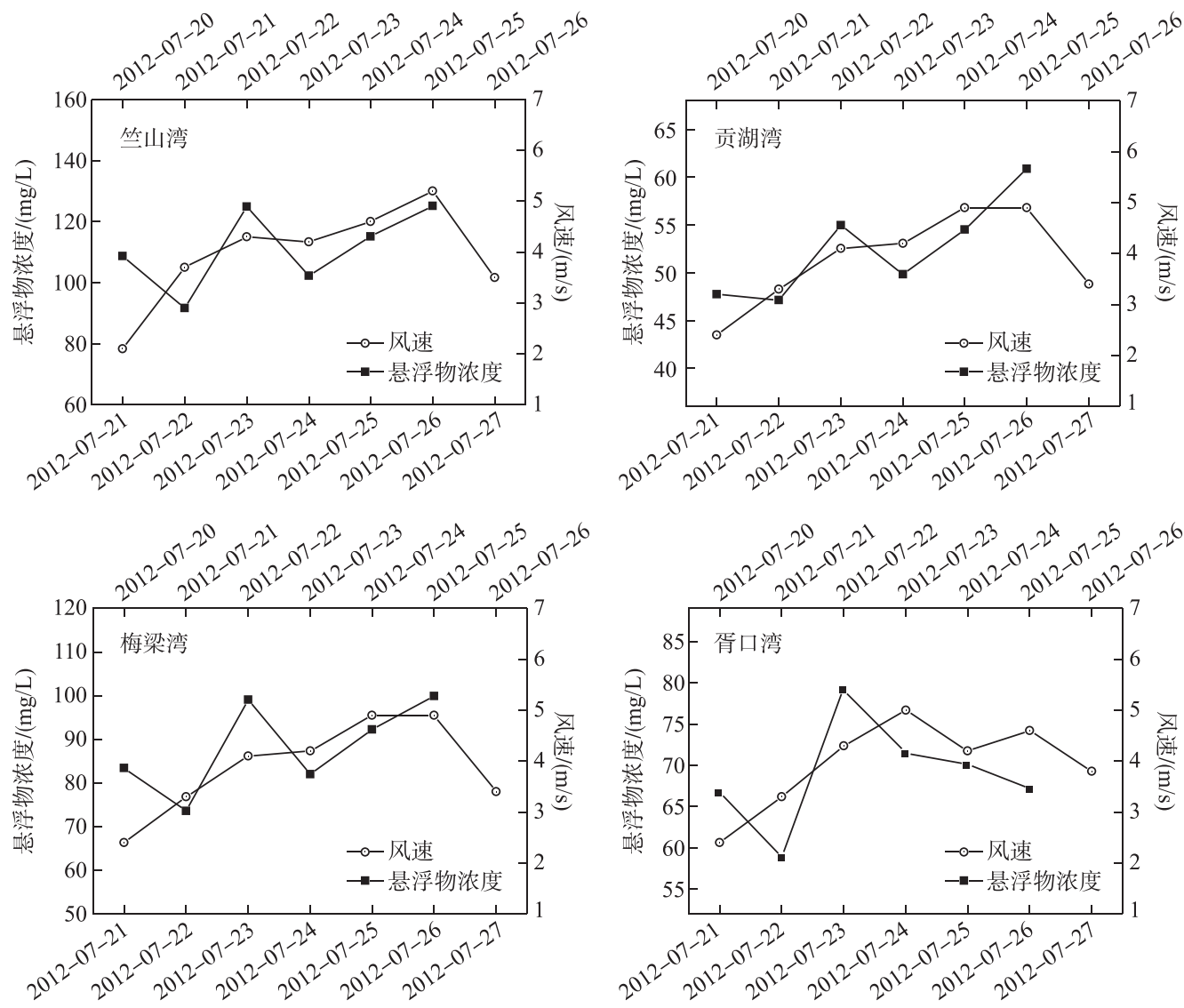

图 9 太湖 4 个湾区悬浮物浓度与风速变化

Fig.9 Corresponding relationships between TSM concentration and wind speeds for the four sub-regions of Lake Taihu

2012 年 7 月 $21-23$ 日太湖悬浮物浓度大于 $50 \mathrm{mg} / \mathrm{L}$ 的区域由西北方向东南方向逐渐扩大, 悬浮物浓 度介于 $80 \sim 120 \mathrm{mg} / \mathrm{L}$ 的区域范围在 23 日急剧扩大 (图 6). 据宜兴和无锡气象站监测的风速数据显示, 21 日 宜兴站监测的风向为 6 (东南风), 无锡站监测的风向为 10 (西南风); 22 日宜兴站监测的风向为 6 (东南风), 无锡站监测的风向为 9 (西南南风), 由于风向的互相削弱作用, 导致 21 与 22 日太湖西北部竺山湾附近的悬 浮物浓度变化不大, 仅悬浮物浓度在 $50 \sim 60 \mathrm{mg} / \mathrm{L}$ 的区域有所增加. 至 23 日宜兴站、无锡站和东山站监测的 风向分别为 7 (东南南风)、6(东南风) 和 6 (东南风), 风向一致且有互相加强之势, 导致胥口湾附近的悬浮 物浓度增高, 并向东南方向扩散, 整个太湖的西北半部悬浮物浓度均大于 $50 \mathrm{mg} / \mathrm{L}$; 至 24 日, 没有了风力的 互相增强,太湖悬浮物浓度大于 $80 \mathrm{mg} / \mathrm{L}$ 的范围缩小,但整个太湖的西北半部悬浮物浓度依旧大于 $50 \mathrm{mg} / \mathrm{L}$. 至 25 日, 虽然太湖的平均风速在减小, 据无锡站和宜兴站气象数据显示, 太湖西部及西北部的风速维持在 一个较高的数量级, 太湖西北部的悬浮物浓度上升并向东南方向扩散. 至 26 日, 太湖的平均风速下降至 3.2 $\mathrm{m} / \mathrm{s}$, 但太湖整体的悬浮物浓度相较于 25 日并没有太大变化, 因为悬浮物浓度变化对风浪的响应有一定的 滞后. 有学者利用太湖站近 10 年来各测点的连续观测资料分析了太湖的悬浮物浓度分布规律, 发现太湖湖 
心区域的悬浮物浓度一般高于其他区域 ${ }^{[30]}$, 而本研究中悬浮物浓度较高的区域处于太湖竺山湾及沿岸 (图 6、图 8), 因为太湖的人湖河流基本位于太湖的西部, 逢勇等 ${ }^{[31]}$ 的研究表明太湖悬浮物浓度湾区及沿岸地区 受湖流的影响较大, 河流悬浮物的汇人、藻颗粒的堆积以及人河口底泥的扰动导致太湖西部区域悬浮物浓 度较高; 而太湖的东部区域浓度较低, 因为此区域为太湖深水区域, 且湖底有大量沉水植被, 在风浪扰动下 极难发生沉积物再悬浮.

本研究利用连续多日逐时高频次的 GOCI 影像数据精细刻画了典型风浪过程影响下的悬浮物短期动态 变化特征并揭示其驱动力, 很好地弥补了 Landsat、MODIS 和 MERIS 等遥感影像数据在时间分辨率上的不 足 $^{[6-7]}$. 本文构建的基于 GOCI 影像数据的遥感估算模型除了用于监测风浪扰动引起的悬浮物浓度短期快速 变化外, 未来也可以与 MODIS 数据相互补充, 用于精细化跟踪强降水事件引起的外源河流输人对近岸水体 悬浮物浓度短期动态变化的影响 ${ }^{[32]}$.

\section{4 结论}

1) 通过对多光谱变量与悬浮物浓度进行相关性分析, 发现 GOCI 的近红外波段(第 7 和第 8 波段) 为太 湖悬浮物浓度估算的敏感波段, 由此构建了基于 GOCI 第 7 波段悬浮物遥感估算单波段经验模型,能较为准 确的估算太湖的悬浮物浓度, 独立验证样本的相对均方根误差为 $28.3 \%$, 平均绝对百分误差为 $24.4 \%$.

2) 通过对 2012 年 7月 21-26日这一风浪过程进行研究, 发现风向风速是影响悬浮物浓度变化的重要 因素; 悬浮物浓度的变化对风浪扰动的响应有一定的滞后性, 滞后时间为数小时到 1 天, 沉积物再悬浮速度 大于沉降速度的临界风速约为 $3.4 \mathrm{~m} / \mathrm{s}$.

3 ) 太湖水体悬浮物浓度存在明显的日内变化,2012 年 7 月 21 日太湖悬浮物浓度西北部较高, 并呈现出 从早至晚递减的趋势.

致谢: 野外观测和气象数据的获取得到太湖湖泊生态系统研究站的领导及工作人员的支持和帮助, 邓瑜兵、 夏忠、白杨等参加野外观测和室内分析, 一并表示感谢.

\section{5 参考文献}

[ 1 ] Cole BE, Cloern JE. An empirical model for estimating phytoplankton productivity in estuaries. Marine Ecology Progress, 1987, 36(3) : 299-305. DOI: 10.3354/meps036299.

[ 2 ] Zhang Y, Wu Z, Liu M et al. Thermal structure and response to long-term climatic changes in Lake Qiandaohu, a deep subtropical reservoir in China. Limnology and Oceanography, 2014, 59(4): 1193-1202. DOI: 10.4319/lo. 2014.59. 4.1193.

[ 3 ] Rickson RJ. Can control of soil erosion mitigate water pollution by sediments? Science of the Total Environment, 2014, 468469(2) : 1187. DOI: 10.1016/j.scitotenv.2013.05.057.

[ 4 ] Fabio N. Güttler, Niculescu S, Gohin F. Turbidity retrieval and monitoring of Danube Delta waters using multi-sensor optical remote sensing data: An integrated view from the delta plain lakes to the western-northwestern Black Sea coastal zone. Remote Sensing of Environment, 2013, 132(6) : 86-101. DOI: 10.1016/j.rse.2013.01.009.

[ 5 ] Zhang Y, Zhang Y, Shi K et al. A Landsat 8 OLI-Based, Semianalytical model for estimating the total suspended matter concentration in the slightly turbid Xin'anjiang Reservoir (China). IEEE Journal of Selected Topics in Applied Earth Observations and Remote Sensing, 2016, 9(1) : 398-413. DOI:10.1109/JSTARS.2015.2509469.

[ 6 ] Shi K, Zhang Y, Zhu G et al. Long-term remote monitoring of total suspended matter concentration in Lake Taihu using $250 \mathrm{~m}$ MODIS-Aqua data. Remote Sensing of Environment, 2015, 164(2) : 43-56. DOI: 10.1016/j.rse.2015.02.029.

[ 7 ] Zhang Y, Shi K, Liu X et al. Lake topography and wind waves determining seasonal-spatial dynamics of total suspended matter in turbid Lake Taihu, China: Assessment using long-term high-resolution MERIS data. PLoS One, 2014, 9(5): e98055. DOI: 10.1371/journal.pone.0098055.

[ 8 ] Zheng Z, Li Y, Guo Y et al. Landsat-based long-term monitoring of total suspended matter concentration pattern change in the wet season for Dongting Lake, China. Remote Sensing, 2015, 7 (10) : 13975-13999. DOI: 10.3390/rs71013975.

[ 9 ] Nechad B, Ruddick KG, Park Y. Calibration and validation of a generic multisensor algorithm for mapping of total suspended matter in turbid waters. Remote Sensing of Environment, 2010, 114(4) : 854-866. DOI: 10.1016/j.rse.2009.11.022 . 
[10] Ma RH, Tang JW, Duan HT et al. Progress in lake water color remote sensing. J Lake Sci, 2009, 21(2) : 143-158. DOI: 10.18307/2009.0201. [马荣华, 唐军武, 段洪涛等. 湖泊水色遥感研究进展. 湖泊科学, 2009, 21(2) : 143-158.]

[11] Zhu GW, Qin BQ, Gao G. Vertical distribution of the concentrations of phosphorus and suspended solid in Taihu Lake affected by wind-induced wave. Advances in Water Science, 2004, 15(6) : 775-780. [ 朱广伟, 秦伯强, 高光. 强弱风浪扰 动下太湖的营养盐垂向分布特征. 水科学进展, 2004, 15(6): 775-780.]

[12] Suh AS, Lee H, Kim D et al. KMA geostationary satellite program: COMS (Communication, Ocean and Meteorological Satellite) and its application. 2009.

[13] Kang G, Kang S, Yong S et al. Korea Geostationary Ocean Color Imager (KGOCI). Proc IGARSS, 2004, 1( C) : 3261 3263. DOI: 10.1109/igarss.2004.1370397.

[14] He X, Bai Y, Pan D et al. Using geostationary satellite ocean color data to map the diurnal dynamics of suspended particulate matter in coastal waters. Remote Sensing of Environment, 2013, 133 (12): 225-239. DOI: 10. 1016/j. rse. 2013. 01.023 .

[15] Huang C, Yang H, Zhu AX et al. Evaluation of the Geostationary Ocean Color Imager GOCI to monitor the dynamic characteristics of suspension sediment in Taihu Lake. International Journal of Remote Sensing, 2015, 36(15) : 3859-3874. DOI: $10.1080 / 01431161.2015 .1070323$.

[16] Qin BQ, Yang GJ, Ma JR et al. Dynamics of variability and mechanism of harmful cyanobacteria bloom in Lake Taihu, China. Chinese Science Bulletin, 2016, 61(7) : 759-770. DOI: 10.1360/N972015-00400. [秦伯强, 杨桂军, 马健荣 等. 太湖蓝藻水华“暴发”的动态特征及其机制. 科学通报, 2016, 61(7)：759-770.]

[17] Xu PZ, Qin BQ. Degeneration of ecosystem of lakeside zone around Taihu Lake and planning for its rehabilitation. Water Resources Protection, 2002, (3) : 31-36. [许朋柱, 秦伯强. 太湖湖滨带生态系统退化原因以及恢复与重建设想. 水 资源保护, 2002, (3) : 31-36.]

[18] Huang CC, Li YM, Wang Q et al. Bio-optical retrieval model of suspended particles in Tai Lake using MERIS image. Journal of Infrared and Millimeter Waves, 2012, 31(4) : 367-374. DOI: 10.3724/SP.J.1010.2012.00367. [黄昌春, 李云 梅, 王桥等. 太湖水体悬浮颗粒物生物光学模型及 MERIS 数据反演. 红外与毫米波学报, 2012, 31(4) : 367-374.]

[19] Yang JX, Wang YP, Yang Y. Atmospheric correction model of remote sensing image by regression analysis between elevation/aerosol optical thickness and correction parameters of 6S model. Remote Sensing Technology and Application, 2009, 24(3) : 331-340. [ 杨静学, 王云鹏, 杨勇. 基于高程或气溶胶厚度与 $6 \mathrm{~S}$ 模型校正参数回归方程的遥感图像大气 校正模型. 遥感技术与应用, 2009, 24(3):331-340.]

[20] Jiang S, Li XW, Niu ZC et al. Monitor and evaluation of atmospheric visibility in Taihu area by remote sensing from 1984 to 2015. Environmental Monitoring in China, 2017, 33(5): 176-179. [姜晟, 李旭文, 牛志春等. 1984-2015 年太湖地 区大气能见度变化遥感监测与评价. 中国环境监测, 2017, 33(5) : 176-179.]

[21] Zhao LN, Wang YN, Jin Q et al. Method for estimating the concentration of total suspended matter in lakes based on goci images using a classification system. Acta Ecologica Sinica, 2015, 35 (16) : 5528-5536. DOI: 10.5846/stxb201411152264. [赵丽娜, 王艳楠, 金琦等. 基于 GOCI 影像的湖泊悬浮物浓度分类反演. 生态学报, 2015, 35(16): 5528-5536.]

[22] Choi JK, Park YJ, Bo RL et al. Application of the Geostationary Ocean Color Imager (GOCI) to mapping the temporal dynamics of coastal water turbidity. Remote Sensing of Environment, 2014, 146: 24-35. DOI:10.1016/j.rse.2013.05.032.

[23] Zhu GW, Qin BQ, Gao G. Direct evidence of phosphorus outbreak release from sediment to overlying water in a large shallow lake caused by strong wind wave disturbance. Chinese Science Bulletin, 2005, 50(1):66-71. [朱广伟, 秦伯强, 高 光. 风浪扰动引起大型浅水湖泊内源磷暴发性释放的直接证据. 科学通报, 2005, 50(1) : 66-71.]

[24] Dobrynin M, Gayer G, Pleskachevsky A et al. Effect of waves and currents on the dynamics and seasonal variations of suspended particulate matter in the North Sea. Journal of Marine Systems, 2010, 82(1/2) : 1-20. DOI: 10.1016/j.jmarsys. 2010.02.012.

[25] Xiang J, Pang Y, Li YP et al. Hydrostatic settling suspended matter of large shallow lake. Advances in Water Science, 2008, 19 (1) : 111-115. [ 向军, 逢勇, 李一平等. 浅水湖泊水体中不同颗粒悬浮物静沉降规律研究. 水科学进展, $2008,19(1): 111-115$.

[26] Qin B, Hu W, Gao G et al. Dynamics of sediment resuspension and the conceptual schema of nutrient release in the large shallow Lake Taihu, China. Chinese Science Bulletin, 2004, 49(1) : 54-64. DOI:10.1007/BF02901743. 
[27] Li Y, Tang C, Wang J et al. Effect of wave-current interactions on sediment resuspension in large shallow Lake Taihu, China. Environmental Science and Pollution Research, 2017, 24(4) : 4029-4039. DOI: 10.1007/s11356-016-8165-0.

[28] Wang J, Pang Y, Li Y et al. Experimental study of wind-induced sediment suspension and nutrient release in Meiliang Bay of Lake Taihu, China. Environmental Science and Pollution Research International, 2015, 22(14) : 10471-10479. DOI: 10.1007/s11356-015-4247-7.

[29] Zheng SS, Wang PF, Wang C et al. Sediment resuspension under action of wind in Taihu Lake, China. International Journal of Sediment Research, 2015, 30(1) : 48-62. DOI:10.1016/S1001-6279(15)60005-1.

[30] Zhang YL, Qin BQ, Chen WM et al. A study on total suspended matter in Lake Taihu. Resources and Environment in the Yangtze Basin, 2004, 13(3) : 266-271. [张运林, 秦伯强, 陈伟民等. 太湖水体中悬浮物研究. 长江流域资源与环 境, 2004, 13(3): 266-271.]

[31] Pang Y, Li YP, Luo LC. Study on the simulation of transparency of Lake Taihu under different hydrodynamic conditions. Science in China, 2005, 35(z2) : 145-156. [逢勇, 李一平, 罗潋苾. 水动力条件下太湖透明度模拟研究. 中国科 学, 2005, 35(z2): 145-156.]

[32] Zhang YL, Shi K, Zhou YQ et al. Monitoring the river plume induced by heavy rainfall events in large, shallow, Lake Taihu using MODIS $250 \mathrm{~m}$ imagery. Remote Sensing of Environment, 2016, 173: 109-121. 